\title{
Experimental observation of the Anderson transition with atomic matter waves
}

\author{
Julien Chabé, ${ }^{1}$ Gabriel Lemarié, ${ }^{2}$ Benoît Grémaud, ${ }^{2}$ Dominique \\ Delande, ${ }^{2}$ Pascal Szriftgiser, ${ }^{1}$ and Jean Claude Garreau ${ }^{1}$ \\ ${ }^{1}$ Laboratoire de Physique des Lasers, Atomes et Molécules, \\ Université des Sciences et Technologies de Lille, \\ CNRS; CERLA; F-59655 Villeneuve d'Ascq Cedex, France* \\ ${ }^{2}$ Laboratoire Kastler Brossel, Université Pierre et Marie Curie, 4 Place Jussieu, F-75005 Paris, France
}

(Dated: October 23, 2018)

\begin{abstract}
We realize experimentally an atom-optics quantum chaotic system, the quasiperiodic kicked rotor, which is equivalent to a 3D disordered system, that allow us to demonstrate the Anderson metalinsulator transition. Sensitive measurements of the atomic wavefunction dynamics and the use of finite-size scaling techniques make it possible to extract both the critical parameters and the critical exponent of the transition, which is in good agreement with the value obtained in numerical simulations of the 3D Anderson model.

PACS numbers: 03.75.-b, 72.15.Rn, 05.45.Mt, 64.70.Tg
\end{abstract}

The metal-insulator Anderson transition plays a central role in the study of quantum disordered systems. An insulator is associated with localized states of the system, while a metal generally displays diffusive transport associated with delocalized states. The Anderson model [1] describes such a metal-insulator transition, due to quantum interference effects driven by the amount of disorder in the system. Starting from the "tight-binding" description of an electron in a crystal lattice, Anderson postulated in 1958 that the dominant effect of impurities in the lattice is to randomize the diagonal, on-site, term of the Hamiltonian, and showed that this generally leads to a localization of the wavefunction, in sharp contrast with the Bloch-wave solution for a perfect crystal. This model has progressively been extended from its original solidstate physics scope [1, 2, 3, 4] to a whole class of systems in which waves propagate in a disordered medium, as for example quantum-chaotic systems [5, 6] and electromagnetic radiation 7, 8, 9]. However mathematically simple, the model predicts a wealth of interesting phenomena. In $1 \mathrm{D}$, the wavefunction is always localized as recently observed in experiments using atomic matter waves in a disordered optical potential [10, 11]; in 3D it predicts a phase transition between a localized (insulator) and a delocalized (metal) phase at a well defined mobility edge, the density of impurities or the energy being the control parameter.

Despite the wide interest on the Anderson transition, few experimental results are available. In a crystal, it is very difficult to obtain the conditions for a clean observation of the Anderson localization. Firstly, one has no direct access to the electronic wavefunction and must rely on modifications of bulk properties like conductivity [2, 12]. Secondly, it is difficult to reduce decoherence sources to a low enough level. We thus engineered a matter-wave system that is described by an Andersonlike model, which allows us to probe the physics of disordered systems in much better conditions than in con- densed matter physics [13], namely: Almost no interaction between particles, weak absorption in the medium, no coupling with a thermal reservoir which could destroy localization and possibility of measuring the final quantum state of the system after a given interaction time. The system, the quasi-periodic kicked rotor [5, 6, 14, 15], consists of cold cesium atoms exposed to a pulsed, offresonant, laser standing wave. The dynamics is thus effectively one-dimensional along the axis of the laser beam, as transverse directions are uncoupled. The atoms interact periodically with the spatially sinusoidal potential whose amplitude is modulated at (incommensurable) frequencies $\omega_{2}$ and $\omega_{3}$, and the corresponding Hamiltonian is:

$H=\frac{p^{2}}{2}+K \cos x\left[1+\varepsilon \cos \left(\omega_{2} t\right) \cos \left(\omega_{3} t\right)\right] \sum_{n=0}^{N-1} \delta(t-n)$,

where $x$ is the particle position (along the laser axis), $p$ is its momentum, and $K$ is the pulse intensity. The pulses are short enough (compared to the center of mass dynamics) to be consider as instantaneous kicks. We have chosen normalized variables such that $x$ is measured in units of the spatial period of the potential divided by $2 \pi$, the particle's mass is unity and time is measured in units of pulse period $T_{1}$.

By taking $\varepsilon=0$ in Eq. (1) one obtains the standard (strictly time-periodic) kicked rotor, a system known to display the phenomenon of dynamical localization [5], which manifests itself by an exponential localization of the wavefunction in momentum space. Dynamical localization exists only when the classical dynamics of the kicked rotor is a chaotic diffusion. In the quantum case, diffusion is inhibited after some localization time by quantum interferences. Dynamical localization has been shown to be a direct analogue of Anderson localization in one dimension [6], with the following correspondences: Localization takes place in real space for the Anderson 


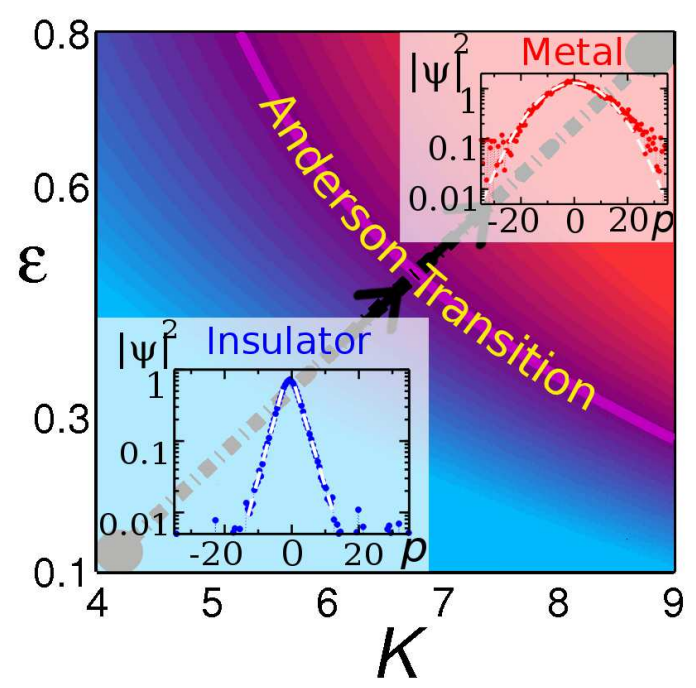

Figure 1: Phase diagram of the quasiperiodic kicked rotor, from numerical simulations. The localized (insulator) region is shown in blue, the diffusive (metallic) region is shown in red. The experimental parameters are swept along the diagonal dash-dotted line. The insets show the experimentally observed momentum distributions, localized in the insulator region and Gaussian in the diffusive (metallic) region.

model and in momentum space for the kicked rotor; the "stochasticity parameter" $K$ [see Eq. (10] corresponds to the ratio of hopping to diagonal energy in the Anderson model. The strictly random disorder in the Anderson model correspond to a pseudo-random potential in the quasi-periodic kicked rotor. Thus, the experimental observation of dynamical localization in the kicked rotor [14] is actually the first observation of Anderson 1D localization with atomic matter waves.

In order to observe the Anderson transition, one must generalize the kicked rotor to obtain a system analogous to the 3D Anderson model. This can be done by introducing further temporal dependencies that make the Hamiltonian quasi-periodic in time, that is to take $\varepsilon \neq 0$ in Eq. (1) with $\omega_{2}$ and $\omega_{3}$ incommensurate numbers. The resulting system has been shown to be substantially equivalent to the 3D Anderson model [15], the additional spatial coordinates in the Anderson model corresponding to the additional time dependencies in the quasi-periodic kicked rotor. The parameters controlling the dynamics are $\omega_{2}$ and $\omega_{3}$ and, more importantly, the kick strength $K$ and the modulation amplitude $\varepsilon$. In the $(K, \varepsilon)$ plane, the $\varepsilon=0$ line corresponds to dynamically localized dynamics (the periodic kicked rotor equivalent to the 1D Anderson model) and the Anderson transition takes place along a critical line in the plane $\varepsilon>0$, as shown in Fig. 1

Our atom-optics realization of the kicked rotor has been described in detail elsewhere [16, 17, 18]. Basically, we cool cesium atoms in a standard magneto-optical trap, and, after a Sisyphus-molasses phase, we obtain a cloud of $10^{7}$ atoms at a temperature of $3.2 \mu \mathrm{K}$. This prepares a sample of atoms in a thermal state whose momentum distribution is much narrower than the expected localization length. The atoms then interact with the optical potential generated by a horizontal standing wave. An acousto-optical modulator driven by an arbitrary-form synthesizer modulates the amplitude of the optical potential. One generates in this way $0.9 \mu$ s-long pulses at $T_{1}=27.778 \mu \mathrm{s}$ (corresponding to an effective Planck constant $k=2.89)$, to which is superimposed a modulation of the form Eq. (10), with $\omega_{2} / 2 \pi=\sqrt{5}$ and $\omega_{3} / 2 \pi=\sqrt{13}$. The standing wave, of typical power $160 \mathrm{~mW}$, is far offresonant $(7.3 \mathrm{GHz}$ to red of the atomic transition, corresponding to $1.4 \times 10^{3}$ natural widths), in order to reduce spontaneous emission. Stimulated Raman transitions allow sensitive measurements of the atomic momentum distribution [17].

The experimental values of the parameters are chosen according to the following considerations. Firstly, in order to prevent classical (KAM barrier) localization effects one must have $K>2[19]$. For all the data presented in this paper, we numerically checked that the classical dynamics is fully diffusive, which excludes that the observed transition could have a classical origin. Secondly, in order to confine the transition to a relatively narrow range of parameters one must cross the critical curve (Fig. 1) "at a right angle"; we thus vary simultaneously the $K$ and $\varepsilon$ along a line going from $K=4, \varepsilon=0.1$ in the localized region to $K=9, \varepsilon=0.8$ in the diffusive region; the critical line is then crossed at $K=K_{c}=6.6$. Thirdly, short enough pulses must be used, so that they can be considered as delta pulses. Numerical simulations taking into account the finite pulse duration $(0.9 \mu \mathrm{s})$ show that less than $1 \%$ of the atoms are sensitive to the duration of the pulses. Finally, decoherence processes must be kept as small as possible. We have identified two dominant decoherence sources in our experiment: i) Spontaneous emission, which is not included in the Hamiltonian Eq. (11) and ii) the deviation of the standing wave from strict horizontality, which mixes quasimomentum classes and produces a stray momentum diffusion. The fact that numerical simulations of the "pure" quasi-periodic kicked rotor shown hereafter agree very well with the experimental results (in particular for the position of the Anderson transition and for the critical exponent) proves that spurious effects are well under control. Moreover, we have checked that inclusion of these effects in the numerical calculations only leads to small changes for $t \leq 150$ kicks.

In order to observe the Anderson transition we apply a sequence of kicks to the atomic cloud and measure its dynamics. In the localized regime, the evolution of its momentum distribution is "frozen" after the localization time $(\sim 12$ kicks $)$ into an exponential curve $\exp \left(-|p| / p_{\text {loc }}\right)$ (where $p_{\text {loc }}$ is the localization length). In the diffusive regime, the initial Gaussian shape is preserved and the distribution gets broader as kicks are ap- 


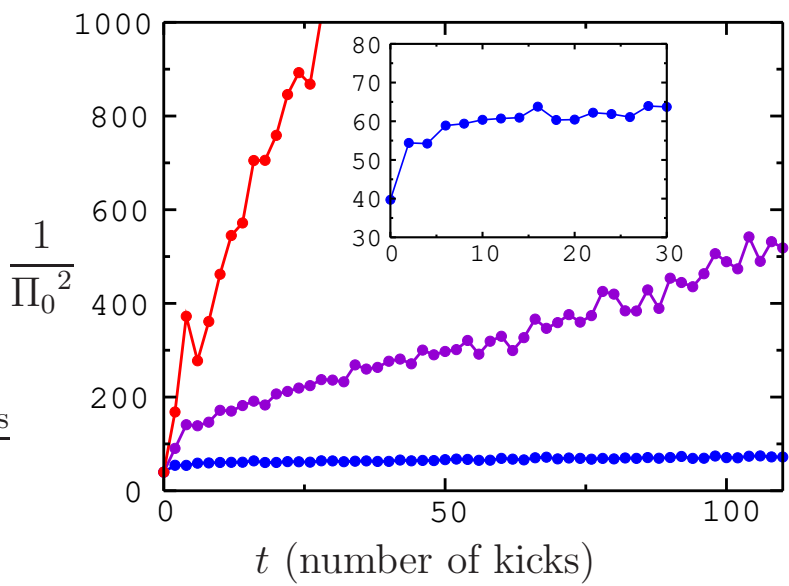

Figure 2: Temporal dynamics of the quasi-periodically kicked rotor. We measure the population $\Pi_{0}(t)$ of the zeromomentum class as a function of time and plotted the quantity $\Pi_{0}^{-2}(t)$ (proportional to $\left\langle p^{2}\right\rangle(t)$ ). Clearly, it tends to a constant in the localized regime (blue curve) and increases linearly with time in the diffusive regime (red curve). Close to the critical point (purple curve), it displays anomalous diffusion $\Pi_{0}^{-2}(t) \sim t^{2 / 3}$, as expected from theoretical arguments [24].

plied, corresponding to a linear increase of the average kinetic energy. The insets in Fig. 1 1 show the experimentally observed momentum distributions, an exponentially localized distribution for $K<K_{c}$ (blue curve), characteristic of dynamical localization, and a broad, Gaussianshaped distribution for $K>K_{c}$ (red curve), characteristic of the diffusive regime. Instead of measuring the full momentum distribution, it is sufficient, and much easier, to measure the population $\Pi_{0}(t)$ of the zero velocity class 21], as $\Pi_{0}^{-2}(t)$ is proportional to $\left\langle p^{2}\right\rangle(t)$ (by virtue of the constancy of the total number of atoms). We performed several experimental runs corresponding to points in the $(K, \epsilon)$ plane. In each run the value of $\Pi_{0}(t)$ was recorded as the kicks were applied. We also recorded the background signal obtained by not applying the Raman detection sequence, and the total number of atoms in the cold-atom cloud. These signals are used to correct the experimental data from background signals and long-term drifts of the cloud population. Figure 2 shows the experimentally observed $\Pi_{0}^{-2}(t)$ and clearly shows the transition from the localized to the diffusive regime, with an intermediate regime of anomalous diffusion.

When one approaches the critical point from the insulator side, the localization length diverges, whereas, on the metallic side, the diffusion constant vanishes. However, a strict divergence can be observed only in macroscopically large samples; in small samples the divergence is smoothed. This fact plagued the numerical studies of the solid-state Anderson transition, as only a finite (small) lattice can be dealt with in a computer. In our system, a singular behavior would show up only for prohibitively large numbers of kicks, which are, in practice, limited to 150 . To overcome this limitation, a technique named "finite-size scaling" 12, 22, 23] was introduced, whose basic idea is to infer the scaling law allowing proper extrapolation of the measured localization length to an infinite sample. We will next show that our data obey the scaling laws predicted by renormalization theory, and this will allow us to extract the critical exponent associated with the singular behavior at the transition.

We have adapted the standard finite-size scaling approach used in numerical studies of the Anderson transition [3] assuming that, for finite interaction time, the quantity $\Lambda(t)=\Pi_{0}^{-2}(t) t^{-2 / 3}$ is an arbitrary function $f\left(\xi t^{-1 / 3}\right)[20,24,25]$, with a scaling parameter $\xi$ which depends only on $K$. Using the results obtained for various values of $t$ and $K$ one can reconstruct both the function $f$ and the scaling parameter $\xi(K)$ (no assumption on the form of $f$ is made). The result is shown in Fig. B(a) and (b) for numerical simulations and in Fig. B(c) and (d) for the experimental results. In both cases, the scaling hypothesis is justified by the fact that all points in Fig. 3(a) and 3(c) lie on a single curve (within the experimental error). The remarkable feature is the existence of two branches: the upper one corresponds to diffusive motion while the lower one corresponds to the localized regime, the critical point being at the tip joining the two branches. The scaling parameter $\xi(K)$ is plotted in Figs. 3(b) and 3(d): It represents the localization length in the localized regime and scales as the inverse of the diffusion constant in the diffusive regime. Clearly, it increases rapidly in the vicinity of the critical value $K_{c}$, on both sides of the transition, and it is found to behave as $\xi \sim\left|K-K_{c}\right|^{-\nu}$ when $K \rightarrow K_{c}$. This divergence at the critical point is a key property of the Anderson phase transition. However, phase-breaking mechanisms induced by decoherence processes smooth the algebraic divergence. We model such effects by introducing a small cutoff of the divergence due to the residual decoherence:

$$
\frac{1}{\xi(K)}=\alpha\left|K-K_{c}\right|^{\nu}+\beta
$$

The experimental observations and the numerical data have been fitted with this formula [dashed curves in Figs. 3(b) and 3(d)]. We found $K_{c}=6.4$ (very close to the value $K_{c}=6.6$ obtained from the numerical simulation), and a critical exponent $\nu=1.4 \pm 0.3$ [26], which is consistent with the numerical value within the error bars. The good agreement between the numerical simulations and the experimental results proves that spurious effects (such as decoherence) are under control. We emphasize that there are no adjustable parameters in our procedure, all parameters are determined using the atoms themselves as probes. Once the existence of the scaling law established, it is more convenient to use a global analysis of the numerical data at various values of $K$ (see refs. 

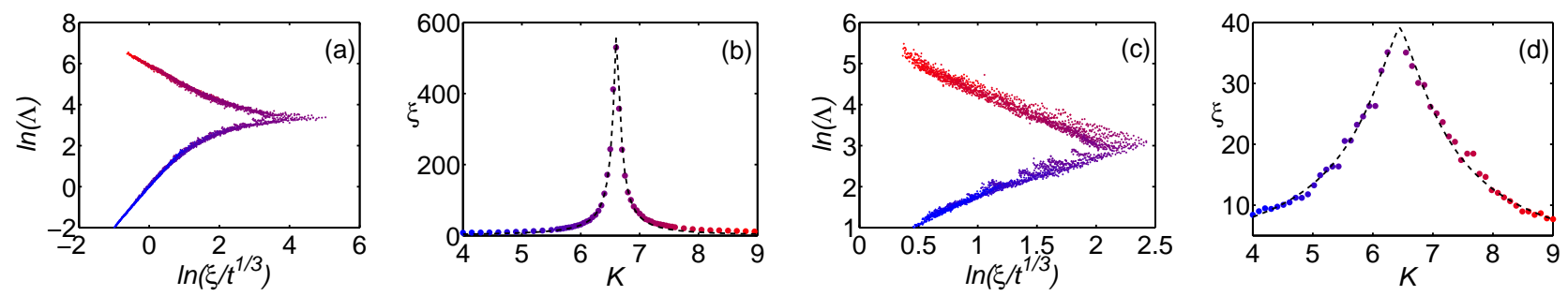

Figure 3: Finite size scaling applied to the results of numerical simulations $[(\mathrm{a})$ and (b)] and to the experimental results [(c) and (d)]. The rescaled quantity $\Lambda=\Pi_{0}^{-2}(t) t^{-2 / 3}$ is plotted as a function of time (from 30 to $10^{4}$ kicks (simulation) and from 30 to 150 kicks (experiment), for various values of $K$. Finite-size scaling of the raw data makes it possible to determine both the scaling function $f$, shown in (a) and (c), and the scaling parameter $\xi(K)$, represented in (b) and (d). Close to $K_{c}$, the behavior of $\xi$ in (b) and (d) is well fitted by Eq. (2) (dashed lines), giving $K_{c}=6.6$ (simulation) and $K_{c}=6.4$ (experiment). The critical exponent is $\nu=1.60 \pm 0.05$ (simulation) and $\nu=1.4 \pm 0.3$ (experiment).

Mackinnon [12], Slevin and Ohtsuki [23]). This, together with the fact that numerical simulations are not limited to 150 kicks and can be ran up to several thousands kicks, make it possible to obtain a more precise numerical value for the critical exponent $\nu=1.60 \pm 0.05$. Note, finally, that although the data displayed here concerns a particular set of parameters we also verified experimentally the presence of the transition for other parameters.

In conclusion, we have presented the first experimental evidence of the Anderson transition with atomic matter waves. The transition is characterized by a well defined critical point, a divergence of the localization length below the critical point (in the localized regime) and a vanishing of the diffusion constant above the critical point (in the diffusive regime). We have determined the scaling laws and the critical exponent $\nu$ of the Anderson transition, which is significantly larger than unity and very close to the value obtained in recent numerical experiments 12, 23] on the Anderson model, enforcing the assumption 15] that the two systems are substantially equivalent. Whether this exponent is universal (i.e. independent of the microscopic details) or not remains to be studied. A very interesting point is that our Andersonequivalent quasiperiodic kicked rotor can be easily generalized to higher dimensions simply by adding new incommensurable frequency, which opens perspectives for fascinating studies of the dependence of the critical exponent on the dimension of the underlying Anderson model.

The authors acknowledge D. Shepelyansky for bringing ref. [15] to their attention, and for fruitful discussions. They also acknowledge G. Beck for his help with the experiment.

* URL: www.phlam.univ-lille1.fr/atfr/cq

[1] P. W. Anderson, Phys. Rev. 109, 1492 (1958).

[2] D. M. Basko, I. L. Aleiner, and B. L. Altshuler, Ann. Phys. 321, 1126 (2006).

[3] B. Kramer and A. Mackinnon, Rep. Prog. Phys. 56, 1469
(1993).

[4] D. J. Thouless, Phys. Rep. 13, 93 (1974).

[5] G. Casati, B. V. Chirikov, J. Ford, and F. M. Izrailev, Stochastic behavior of a quantum pendulum under periodic perturbation (Springer-Verlag, Berlin, Germany, 1979), vol. 93, pp. 334-352.

[6] D. R. Grempel, R. E. Prange, and S. Fishman, Phys. Rev. A 29, 1639 (1984).

[7] M. Störzer, P. Gross, C. M. Aegerter, and G. Maret, Phys. Rev. Lett. 96, 063904 (2006).

[8] T. Schwartz, G. Bartal, S. Fishman, and B. Segev, Nature (London) 446, 52 (2007).

[9] C. Dembowski, H. D. Gräf, R. Hofferbert, H. Rehfeld, A. Richter, and T. Weiland, Phys. Rev. E 60, 3942 (1999).

[10] J. Billy, V. Josse, Z. Zuo, A. Bernard, B. Hambrecht, P. Lugan, D. Clément, L. Sanchez-Palencia, P. Bouyer, and A. Aspect, Nature (London) 453, 891 (2008).

[11] G. Roati, C. d'Errico, L. Fallani, M. Fattori, C. Fort, M. Zaccanti, G. Modugno, M. Modugno, and M. Inguscio, Nature (London) 453, 895 (2008).

[12] A. Mackinnon, J. Phys.: Condes. Matter 6, 2511 (1994).

[13] M. Lewenstein, A. Sanpera, V. Ahufinger, B. Damski, A. Sen, and U. Sen, Advances in Physics 56, 243 (2007).

[14] F. L. Moore, J. C. Robinson, C. F. Bharucha, B. Sundaram, and M. G. Raizen, Phys. Rev. Lett. 75, 4598 (1995).

[15] G. Casati, I. Guarneri, and D. L. Shepelyansky, Phys. Rev. Lett. 62, 345 (1989).

[16] P. Szriftgiser, H. Lignier, J. Ringot, J. C. Garreau, and D. Delande, Commun. Nonlin. Sci. Num. Simul. 8, 301 (2003).

[17] J. Ringot, P. Szriftgiser, and J. C. Garreau, Phys. Rev. A 65, 013403 (2001).

[18] J. Ringot, Y. Lecoq, J. C. Garreau, and P. Szriftgiser, Eur. Phys. J. D 7, 285 (1999).

[19] The standard kicked rotor becomes fully chaotic for $K>5$, but our quasi-periodic version is chaotic for much smaller values $K>2$.

[20] F. Wegner, Z. Phys. B25, 327 (1976).

[21] The resolution of the Raman setup is chosen so that the width of this velocity class is 5 normalized momentum units. We experimentally verified that such a resolution produces a broadening of momentum distributions of less than $10 \%$.

[22] M. E. Fisher and M. N. Barber, Phys. Rev. Lett. 28, 
1516 (1972).

[23] K. Slevin and T. Ohtsuki, Phys. Rev. Lett. 82, 382 (1999).

[24] T. Ohtsuki and T. Kawarabayashi, J. Phys. Soc. Jpn. 66, 314 (1997).

[25] D. Stauffer and A. Aharony, Introduction to Percolation
Theory (Taylor and Francis, 1994), 2nd ed.

[26] As is well known from numerical experiments on the Anderson model, the finite-size scaling method slightly underestimates the critical exponents [12]. 